OPEN ACCESS

Edited by:

Filippo Cieri,

Cleveland Clinic, United States

Reviewed by:

Roser Sala-Llonch,

University of Barcelona, Spain

Paola Valsasina,

San Raffaele Scientific Institute

(IRCCS), Italy

*Correspondence:

Zhongxiang Ding

hangzhoudzx73@126.com

Specialty section:

This article was submitted to

Brain Imaging Methods,

a section of the journal

Frontiers in Neuroscience

Received: 29 January 2019 Accepted: 15 April 2019

Published: 07 May 2019

Citation:

Feng $Q$, Wang $M$, Song $Q, W u Z$, Jiang $H$, Pang P, Liao Z, Yu E and

Ding Z (2019) Correlation Between

Hippocampus MRI Radiomic Features and Resting-State Intrahippocampal

Functional Connectivity in Alzheimer's

Disease. Front. Neurosci. 13:435.

doi: 10.3389/fnins.2019.00435

\title{
Correlation Between Hippocampus MRI Radiomic Features and Resting-State Intrahippocampal Functional Connectivity in Alzheimer's Disease
}

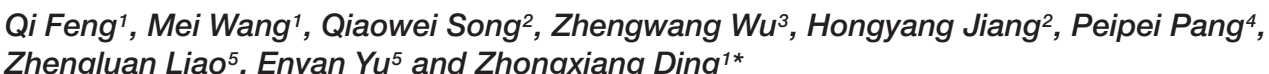

1 Department of Radiology, Affiliated Hangzhou First People's Hospital, Zhejiang University School of Medicine, Hangzhou, China, ${ }^{2}$ Department of Radiology, Zhejiang Provincial People's Hospital, Hangzhou Medical College, Hangzhou, China, ${ }^{3}$ Department of Radiology and BRIC, The University of North Carolina at Chapel Hill, Chapel Hill, NC, United States, ${ }^{4}$ GE Healthcare Life Sciences, Hangzhou, China, ${ }^{5}$ Department of Psychiatry, Zhejiang Provincial People's Hospital, Hangzhou Medical College, Hangzhou, China

Alzheimer's disease (AD) is a neurodegenerative disease with main symptoms of chronic primary memory loss and cognitive impairment. The study aim was to investigate the correlation between intrahippocampal functional connectivity (FC) and MRI radiomic features in AD. A total of $67 \mathrm{AD}$ patients and 44 normal controls (NCs) were enrolled in this study. Using the seed-based method of resting-state functional MRI (rs-fMRI), the whole-brain FC with bilateral hippocampus as seed was performed, and the FC values were extracted from the bilateral hippocampus. We observed that $A D$ patients demonstrated disruptive FC in some brain regions in the left hippocampal functional network, including right gyrus rectus, right anterior cingulate and paracingulate gyri, bilateral precuneus, bilateral angular gyrus, and bilateral middle occipital gyrus. In addition, decreased FC was detected in some brain regions in the right hippocampal functional network, including bilateral anterior cingulate and paracingulate gyri, right dorsolateral superior frontal gyrus, and right precentral gyrus. Bilateral hippocampal radiomics features were calculated and selected using the A.K. software. Finally, Pearson's correlation analyses were conducted between these selected features and the bilateral hippocampal FC values. The results suggested that two gray level runlength matrix (RLM) radiomic features and one gray level co-occurrence matrix (GLCM) radiomic feature weakly associated with $\mathrm{FC}$ values in the left hippocampus. However, there were no significant correlations between radiomic features and FC values in the right hippocampus. These findings present that the AD group showed abnormalities in the bilateral hippocampal functional network. This is a prospective study that revealed the weak correlation between the MRI radiomic features and the intrahippocampal FC in AD patients.

Keywords: Alzheimer's disease, resting-state functional magnetic resonance imaging, functional connectivity, hippocampus, radiomics 


\section{INTRODUCTION}

Alzheimer's disease $(\mathrm{AD})$ is a neurodegenerative disease with main symptoms of chronic primary memory loss and cognitive impairment. $\mathrm{AD}$ has become a public health problem due to its hidden onset, high incidence, and lack of effective drug treatment. The exact cause of AD is still unclear. It is believed some may be related to heredity, neurotransmitter changes, virus infection, immune dysfunction, and free radical damage. Nowadays, the clinical diagnosis of $\mathrm{AD}$ is based on cognitive measures and one or more biomarkers including structural MRI, PET, and cerebrospinal fluid analysis of amyloid $\beta$ or tau proteins (Bruno et al., 2007). Researchers are investigating non-invasive neuroimaging biomarkers for the early diagnosis of AD (Claudia et al., 2010; Yong et al., 2014).

Cell degeneration in the hippocampus plays an important role in the onset of $\mathrm{AD}$. It proved to be the cause of $\mathrm{AD}$ that amyloid- $\beta$ plaques and Tau proteins are selectively deposited in the special cortex of the hippocampus in AD patients (Ball, 1997; Guzman et al., 2013). These special cortexes are the main pathways that connect the hippocampus to the other cortexes of the brain. A large number of magnetic resonance imaging (MRI) studies have revealed atrophy and some other changes in microstructure in the hippocampus in $\mathrm{AD}$ dementia (De et al., 2015; Mak et al., 2017).

MRI is an important technique for radiomics. MR images can acquire numerous sequences and do not receive a radiation dose. MRI shows both the structural change and the functional dynamic change. Several MRI techniques have been applied in $\mathrm{AD}$ research, including voxel-based morphometry (Whitwell et al., 2007), diffusion tensor imaging (DTI) (Denise et al., 2004), resting-state functional MRI (rs-fMRI; Wang et al., 2011), as well as radiomics analysis (Qi et al., 2018). Radiomics is a new frontier subject based on quantitative imaging, feature calculation, feature selection, and model construction. It uses a large number of automated feature extraction algorithms to transform the original image data into first-order or higherorder data, and then analyzes the deep relationship between the data to further improve the accuracy of clinical diagnosis and prognostic value. Radiomics has strong power for radiotherapy, chemotherapy, and immunotherapy evaluation (Horvat et al., 2018; Sun et al., 2018; Wang et al., 2018), cancer patients survival prediction (Kickingereder et al., 2016), molecular subtyping of tumor (Lu et al., 2018), and cancer recurrence prediction (Li H. et al., 2017). Nowadays, radiomics is also applied to non-tumor diseases, such as attention deficit hyperactivity disorder (Port, 2018) and autism spectrum disorder (Chaddad et al., 2017a). In this context, we select the high-resolution T1-weighted MR images for hippocampal microstructural radiomics analysis.

Rs-fMRI is a functional MRI technique that based on blood oxygen level dependent (BOLD), and has emerged as one of the most important techniques for analysis of human brain function. Functional connectivity (FC) is the indirect reflection of synaptic connections. Rs-fMRI FC analysis is increasingly used to detect brain network changes in AD. There are two main methods used in rs-fMRI FC analysis. One is the "seed-based" approach, and the other is independent component analysis (ICA). In seed-based analysis, first, you need to set up a region of interest (ROI), then select BOLD signal fluctuations from the ROI, and associate them with BOLD signal fluctuations for all other voxels in the brain (Sheline and Raichle, 2013). The default mode network (DMN) is most commonly shown to be active when a person is not focused on the outside world and the brain is at wakeful rest, such as during daydreaming and mind-wandering. It is highly correlated with cognitive function (Menon, 2011). FC altered in DMN becomes a potential non-invasive biomarker in diagnosis of $\mathrm{AD}$ and amnestic mild cognitive impairment (aMCI) (Damoiseaux et al., 2012; Toussaint et al., 2014; Yu et al., 2016; Alderson et al., 2017). Functional activity in the hippocampus is the hot topic of $\mathrm{AD}$ research. One study found decreased synchrony of low-frequency fluctuations within the hippocampus in AD patients (Shi-Jiang et al., 2002). An ICA study of fMRI showed that the hippocampal activity diminished in $\mathrm{AD}$ (Wu et al., 2011). Intrinsic connectivity altered in hippocampal functional networks has been observed in aMCI patients (De et al., 2017). However, most of the rs-fMRI studies were focused on the $\mathrm{DMN}$ and some local brain regions; little is known regarding the hippocampus functional network.

Many studies investigated the altered structure or function in the hippocampus in AD patients. Nevertheless, little is known regarding the relationship between hippocampal microstructure and $\mathrm{FC}$ in $\mathrm{AD}$. The hypothesis of the present study is that the local structural change in hippocampus is correlated to the functional changes of the brain in AD patients. The aim of the study was to investigate the correlation between hippocampal MRI radiomic features and intrahippocampal FC in $\mathrm{AD}$, and to explore noninvasive imaging biomarkers for early diagnosis of $\mathrm{AD}$.

\section{MATERIALS AND METHODS}

\section{Study Cohort}

There were $82 \mathrm{AD}$ patients and 50 normal controls (NCs) recruited initially. AD patients were enrolled prospectively in the study at the Zhejiang Provincial People's Hospital from September 2016 to June 2018. The NCs were volunteers collected from the health promotion center of the hospital. All subjects were right-handed and provided written informed consent.

TABLE 1 | Demographics and cognitive characteristics of the participants.

\begin{tabular}{|c|c|c|c|c|}
\hline & AD patients & NC group & Statistic & $P$-value \\
\hline Sample size & 67 & 44 & NA & NA \\
\hline $\begin{array}{l}\text { Age (years, } \\
\text { mean } \pm S D \text { ) }\end{array}$ & $68.75 \pm 11.69$ & $65.48 \pm 9.69$ & 1.54 & 0.13 \\
\hline $\begin{array}{l}\text { Gender } \\
\text { (male/female) }\end{array}$ & $29: 38$ & $20: 24$ & $0.05^{*}$ & $0.82^{*}$ \\
\hline $\begin{array}{l}\text { Education } \\
\text { (years, } \\
\text { mean } \pm \mathrm{SD} \text { ) }\end{array}$ & $6.10 \pm 3.78$ & $7.11 \pm 3.36$ & -1.44 & 0.15 \\
\hline MMSE & $17.16 \pm 5.54$ & $29.09 \pm 0.77$ & -14.17 & $<0.01$ \\
\hline
\end{tabular}

$S D$, standard deviation; MMSE, Mini-Mental State Examination. AD, Alzheimer's disease; NC, normal control. Statistics were calculated with t-tests, unless otherwise indicated. * $x^{2}$-test was used. 


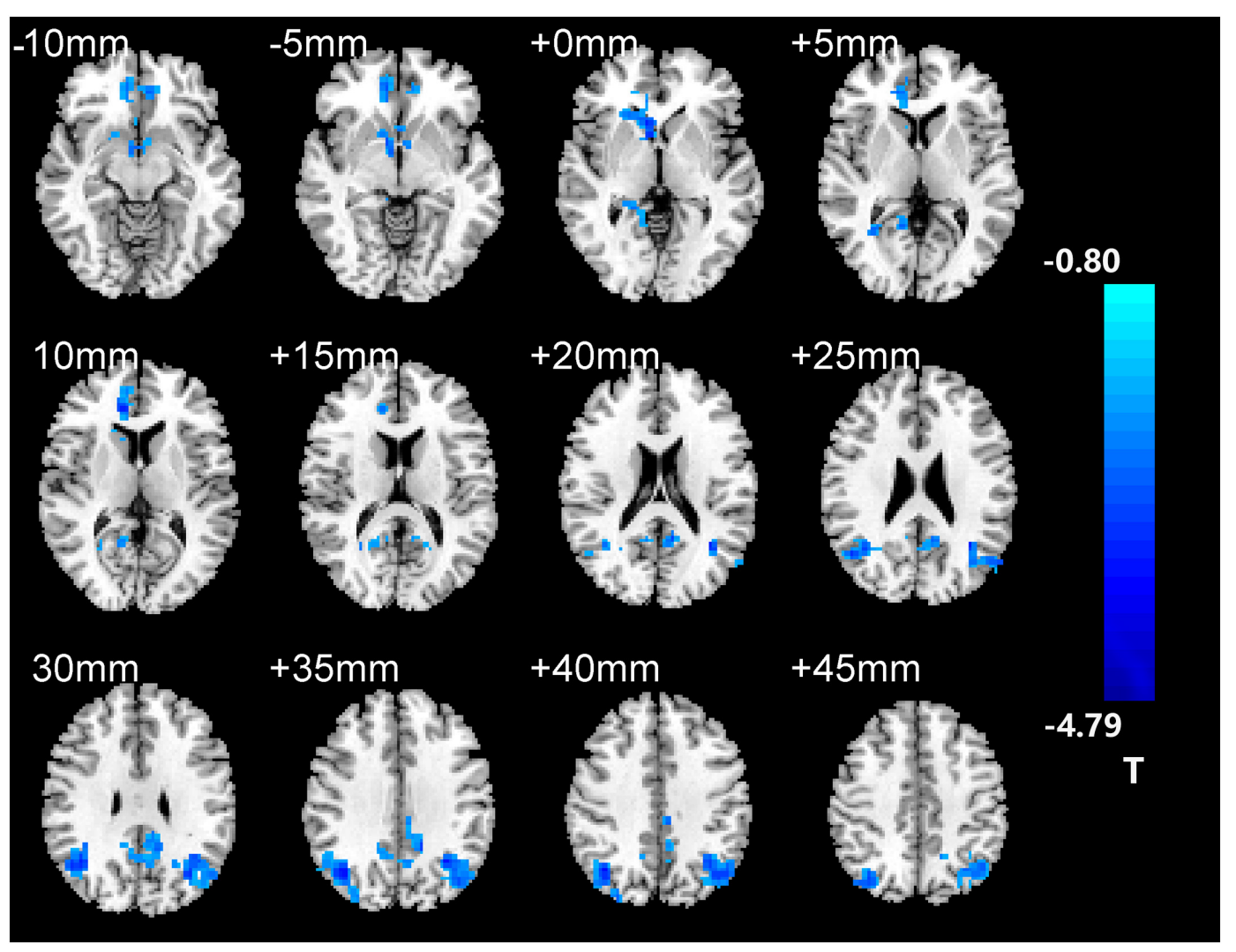

FIGURE 1 | The left hippocampus seed-based functional connectivity (FC) maps in the Alzheimer's disease (AD) patients compared with normal control (NC) subjects.

This study was approved by the Ethics Committee of Zhejiang Provincial People's Hospital (No. 2012KY002) and had been carried out in accordance with the Declaration of Helsinki.

All subjects underwent medical history collection, laboratory examination, neuropsychological test, physical examination, and conventional brain MRI scans. The neuropsychological tests included the Mini-Mental State Examination (MMSE) and Montreal Cognitive Scale (MoCA). AD patients were diagnosed in terms of the revised NINCDS-ADRDA (National Institute of Neurological and Communicative Disorders and Stroke and the Alzheimer's Disease and Related Disorders Association) criteria (Amp, 2011) with MMSE score $\leq 24$ and MoCA score $\leq 26$. The inclusion criteria for NC subjects were as follows: (1) no neurological deficits, such as hearing or vision loss; (2) no neurological or mental diseases, such as stroke, epilepsy, or depression; (3) no evidence of infarction, hemorrhage, or tumor on routine MRI; and (4) MMSE score $\geq 28$. For both AD and NC groups, the exclusion criteria were (1) stroke; (2) brain trauma; (3) brain tumors, Parkinson's disease, epilepsy, and other neurological diseases that cause memory disorders; (4) serious anemia, hypertension, diabetes, and other systemic diseases; (5) history of mental illness; and (6) signal abnormalities in the medial temporal lobe caused by infectious or vascular factors on MRI FLAIR or T2 images (Dubois et al., 2007). There were 73 AD patients and $45 \mathrm{NC}$ subjects who completed all the required MR sequences successfully.

TABLE 2 | Regions showing altered FC for AD patients and NC subjects with the left hippocampus as seed.

\begin{tabular}{|c|c|c|c|c|}
\hline Anatomical region & BA & MNI coordinates $(x, y, z)$ & Cluster size (voxels) & $T$-value \\
\hline REC.R, ACG.R, ORBmid.R, CAU.R & $11 / 10 / 32 / 25$ & $-6, \quad 39,-15$ & 344 & -3.458 \\
\hline PCUN.L, PCUN.R, DCG.L & $31 / 7$ & $-15,-48,33$ & 264 & -3.762 \\
\hline ANG.R, MOG.R & $39 / 19 / 7$ & $39,-66,39$ & 280 & -3.600 \\
\hline ANG.L, MOG.L, IPL.L & $39 / 40 / 19$ & $-33,-60,33$ & 337 & -3.492 \\
\hline
\end{tabular}

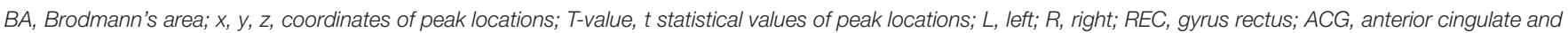

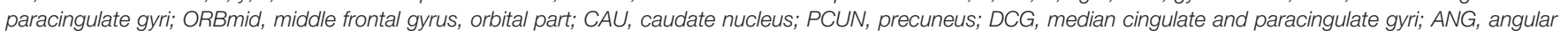
gyrus; MOG, middle occipital gyrus; IPL, inferior parietal but supramarginal and angular gyri. 


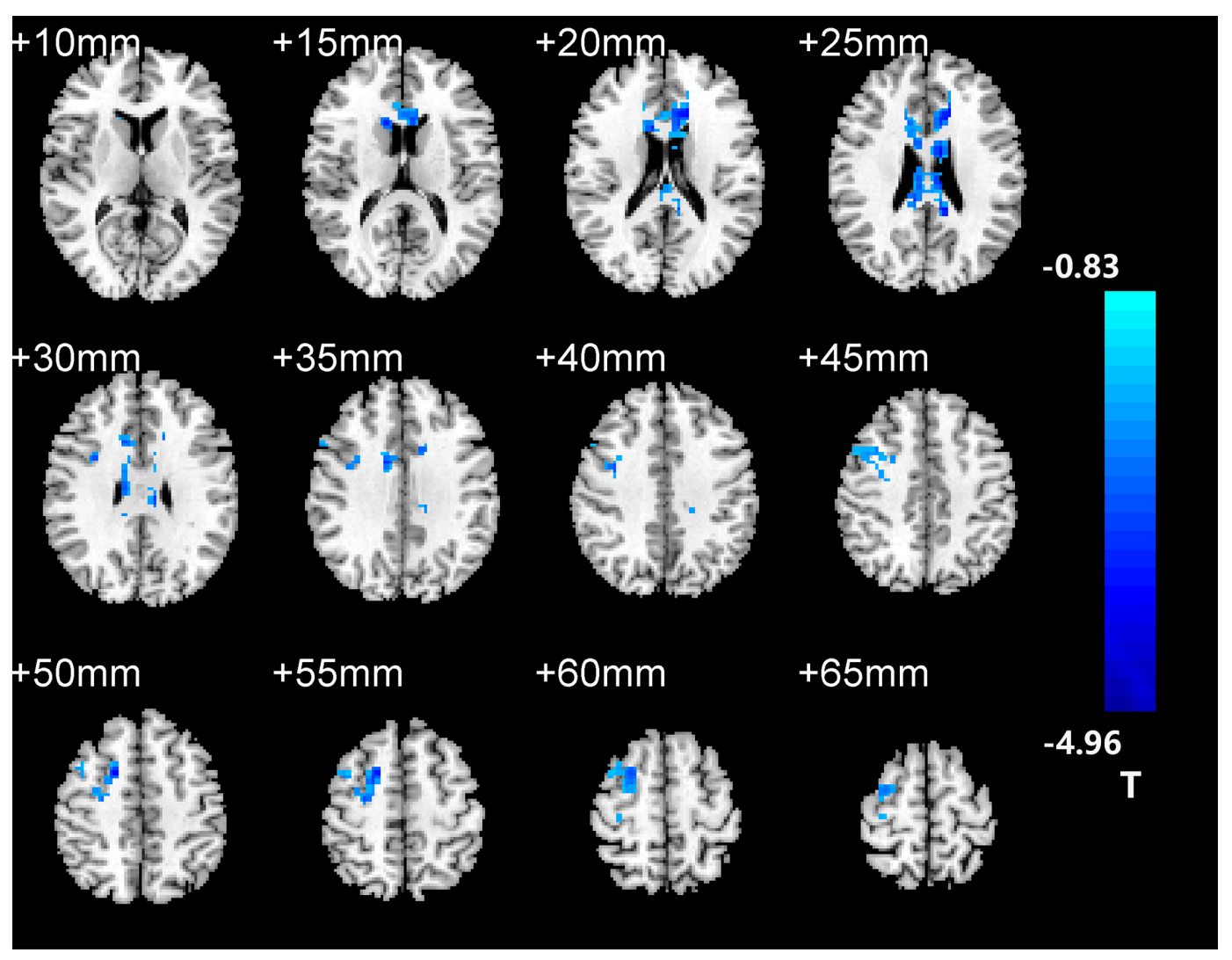

FIGURE 2 | The right hippocampus seed-based FC maps in the AD patients compared with NC subjects.

\section{MRI Acquisition}

Each subject underwent both structural and fMRI examinations on a 3.0-T MR scanner (Discovery MR750; GE Healthcare, Waukesha, WI, United States). The structural MRIs were acquired using a high-resolution three-dimensional T1-weighted magnetization-prepared rapid gradient echo (MPRAGE) sagittal sequence with scanning parameters of repetition time $(\mathrm{TR})=6.7 \mathrm{~ms}$, echo time $(\mathrm{TE})=2.9 \mathrm{~ms}$, inversion time $(\mathrm{TI})=450 \mathrm{~ms}$, slice thickness $/$ gap $=1 / 0 \mathrm{~mm}, \mathrm{FOV}=256 \times 256$ $\mathrm{mm}^{2}$, flip angle $=12^{\circ}$, matrix $=256 \times 256$; there were 192 sagittal slices collected from each subject. The rs-fMRI images were acquired using an echo-planar imaging (EPI) sequence with scanning parameters of $\mathrm{TR}=2,000 \mathrm{~ms}, \mathrm{TE}=30 \mathrm{~ms}$, slice thickness $/$ gap $=3.2 / 0 \mathrm{~mm}, \mathrm{FOV}=220 \times 220 \mathrm{~mm}^{2}$, and flip angle $=90^{\circ}$. Each rs-fMRI sequence contained 210 time points and each time point contained 44 slices. During rs-fMRI, all subjects were instructed to keep still and keep their eyes closed, but to not fall asleep.

\section{Preprocessing of Resting-State Functional MRI}

The image analysis of rs-fMRI after acquisition consists of preprocessing and FC analysis. The preprocessing was performed using the Data Processing Assistant for rs-fMRI (DPARSF ${ }^{1}$ ). The preprocessing steps were as follows:

(1) removing the first 10 time points (considering the time when the magnetic field of the machine was stable and the time when the subject adapted to the environment);

(2) slice timing correction and head motion correction;

\footnotetext{
${ }^{1}$ http://rfmri.org/DPARSF
}

TABLE 3 | Regions showing altered FC for AD patients and NC subjects with the right hippocampus as seed.

\begin{tabular}{|c|c|c|c|c|}
\hline Anatomical region & BA & MNI coordinates $(x, y, z)$ & Cluster size (voxels) & $T$-value \\
\hline ACG.L, ACG.R, DCG.R & $24 / 32 / 23$ & $-9,-21,27$ & 429 & -4.703 \\
\hline SFGdor.R, PreCG.R, MFG.R & $6 / 8$ & $18,6,51$ & 276 & -3.793 \\
\hline
\end{tabular}



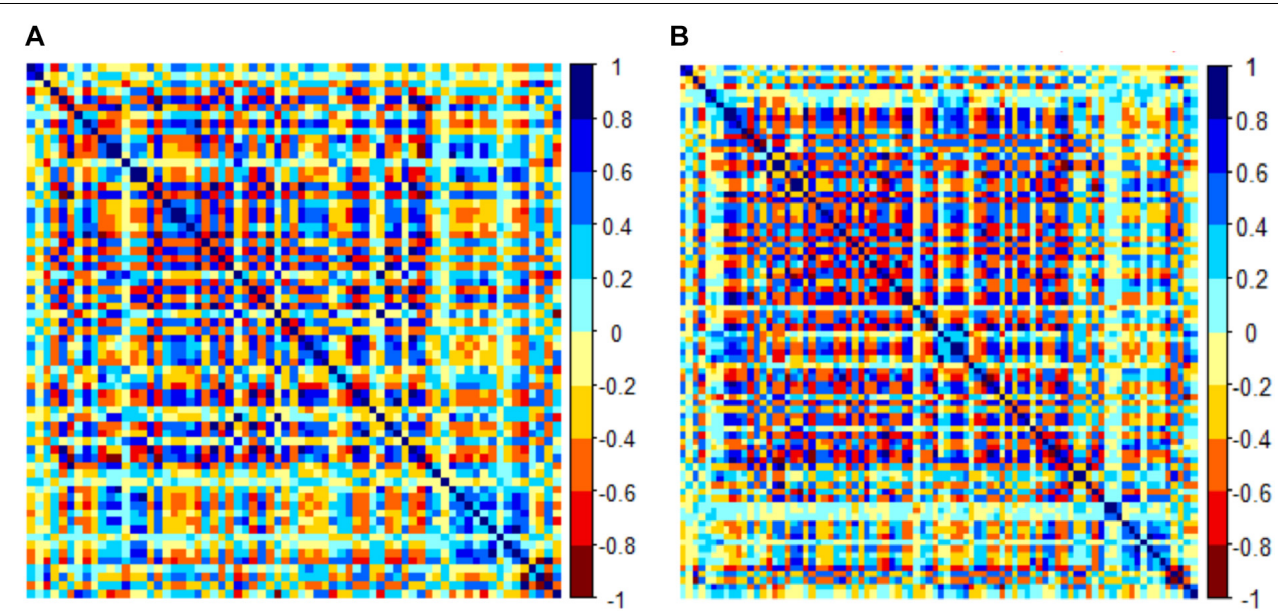

FIGURE 3 | Correlation analysis graph of the left hippocampus (A). Correlation analysis graph of the right hippocampus (B). Each grid represents the feature that enters the correlation analysis after the first step of dimensionality reduction.

(3) normalization to the Montreal Neurological Institute $(\mathrm{MNI})$ space and resampling (with voxels of $3 \times 3 \times 3 \mathrm{~mm}$ );

(4) spatial smoothing using a 4-mm isotropic Gaussian kernel; removing the linear trend; bandpass filtering (0.01$0.08 \mathrm{~Hz}$ ); and

(5) regression of covariates, including the six head motion parameters, the white matter, and cerebrospinal fluid signal.

\section{Segmentation of Hippocampus}

The 3D T1-weighted MPRAGE images were used in the hippocampus segmentation using an efficient learning-based deformable model (Wu et al., 2017). A joint classification and regression model was established to predict the location of the hippocampus. In the training stage, the extracted features were used to train the structured random forest classifier, and in the testing stage, the extracted features were input into the classifier in order to predict the segmentation of each hippocampus, and the prediction segmentation is iteratively improved through the training model. Finally, the hippocampal shape model gradually deformed to the target image to adapt the target hippocampus. We used this method to segment the right and left hippocampus, respectively. The segmentation results had been checked by a senior neuroradiologist, and the data with poor segmentation quality were resegmented.

\section{Resting-State Functional Connectivity Analysis}

The FC analysis of the bilateral hippocampus network was performed using REST plus $\mathrm{V} 1.2^{2}$. We took the averaged time courses of all voxels in ROI (above segmented left and right hippocampus as defined) as the reference sequence. And Pearson's correlation was conducted between the BOLD time course within the ROI and each voxel in the brain. Then, ROI-wise whole-brain FC maps were obtained. Fisher's

${ }^{2}$ http://restfmri.net/forum/RESTplusV1.2 $r$-to- $z$ transformation was used to convert FC maps into normalized zFC maps for subsequent statistical analyses. Onesample $t$-test was conducted in the $\mathrm{AD}$ and $\mathrm{NC}$ groups to make a mask using Gaussian random field (GFR) correction. Two-sample $t$-test was then performed using the above mask between the AD and NC groups. Then, GFR correction was performed (voxel-level $P<0.05$, cluster-level $P<0.05$, twotailed). Finally, the brain areas of significant differences were obtained. Furthermore, in order to quantify the FC values of the bilateral hippocampus in the brain network for next correlation analysis, the mean $Z$-value within the bilateral hippocampus was calculated using the above segmented left and right hippocampus images as ROI definition. The mean $Z$-value represents the average $\mathrm{FC}$ value within the hippocampus. These steps were performed in the left and right hippocampus data, respectively.

\section{MRI Radiomic Analysis}

Artificial Intelligence Kit (A.K) is a commercially available software developed by GE Healthcare Institute of Precision Medicine. It performs data loading, segmentation, feature calculation, feature selection, and model establishment of radiomics.

First, we loaded the original 3D T1-weighted images and ROI images into the A.K. software. Then, image features were calculated including Formfactor, Histogram, Haralick, gray level co-occurrence matrix (GLCM), and gray level runlength matrix (RLM). Formfactor features use mathematical methods to characterize the shape of the lesion, and describe the shape and compactness of the lesion. Histogram features calculate the gray intensity information of the lesion and describe the overall distribution of gray level information. GLCM obtains the co-occurrence matrix by counting the probability of the occurrence of pixel pairs in different directions and displacement vectors. It describes the complexity of the lesion, the level variation, and the degree of texture 

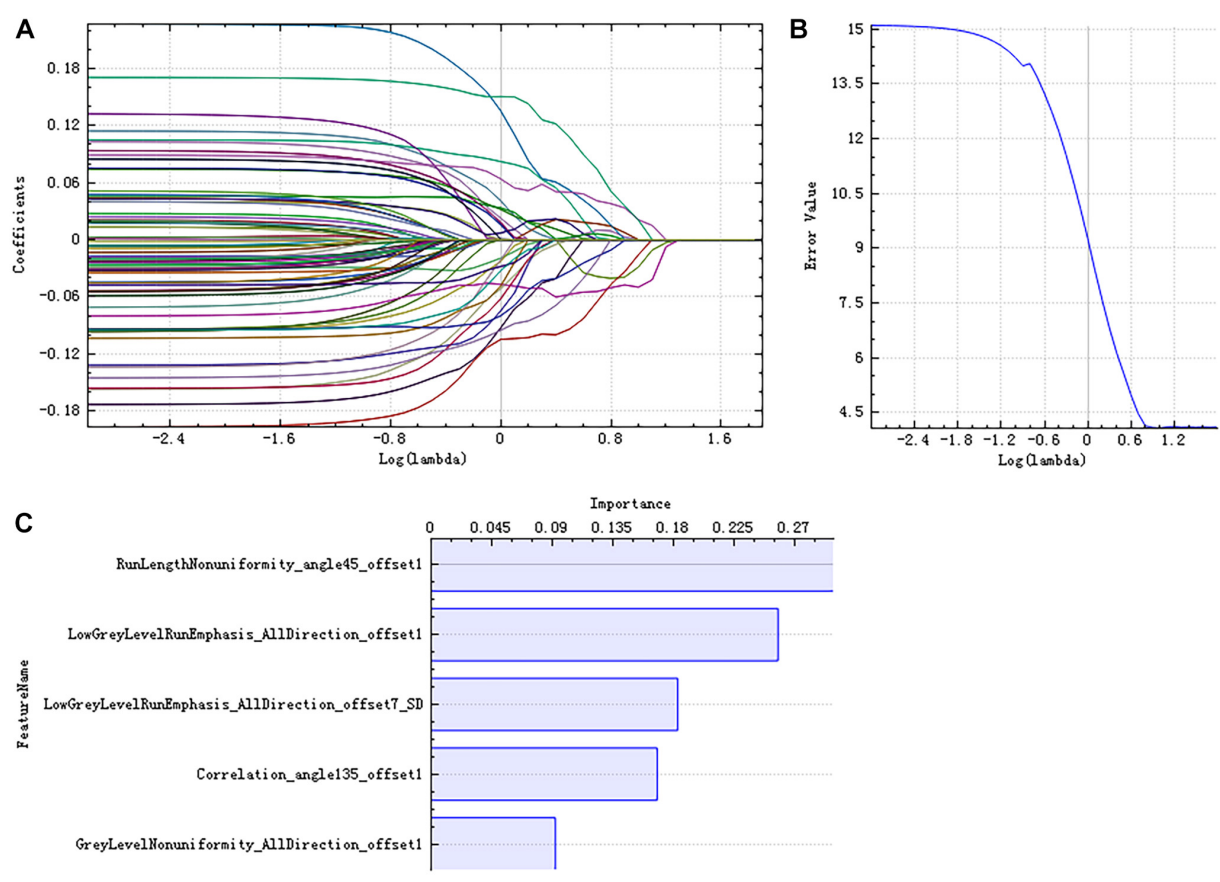

FIGURE 4 | Least absolute shrinkage and selection operator (LASSO) dimensionality reduction of the left hippocampus. Error-lambda graph (A); coefficients-lambda graph (B). We chose $\lambda$ according to the lowest error rate. Importance of the selected features (C).

thickness (Seongin et al., 2011). Haralick also based on the co-occurrence matrix to extract the corresponding features, but it has directional invariance. RLM obtains the length matrix by calculating the probability of the pixels appearing repeatedly in succession with different directions and displacement vectors. It also describes the complexity of the lesion, the level variation and the degree of texture thickness (Galloway, 1975). We chose "1, 4, and 7" for displacement vectors.

After feature calculation, we added labels " 0 " and " 1 " for each subject data representing $\mathrm{NC}$ and $\mathrm{AD}$. The preprocessing for feature selection included replaced the abnormal value with the mean; set the training set proportion and testing set proportion to 0.7 and 0.3 , respectively, and eliminate the unit restriction for each feature column through normalization. The feature selection steps were as follows. Step 1: T-test and rank sum test were used to identify the features with significant differences between the two groups $(P<0.05)$. Step 2: The correlation analysis was used to reduce the dimension. The filter threshold was set to 0.9 , and the Spearman rank correlation coefficient was selected. Correlation analysis was performed between any two feature columns; one of the two highly correlated features was removed when the correlation coefficient was greater than 0.9. Step 3: The least absolute shrinkage and selection operator (LASSO) regression model was applied to reduce the dimension using 10-fold cross-validation. This method is applicable to the regression analysis of high-dimensional data. We performed feature selection on the left and right hippocampus data, respectively.

\section{Statistical Analysis}

Demographic, neuropsychological comparison, RS FC comparison of the two groups, and correlation analysis between the bilateral hippocampus radiomic features and FC were performed using SPSS version 22.0. All statistical methods about radiomic analysis were performed using the A.K. software.

\section{RESULTS}

\section{Comparison of Demographic and Neuropsychological Performance}

Among the remaining $73 \mathrm{AD}$ patients and $45 \mathrm{NC}$ subjects, $67 \mathrm{AD}$ patients and $44 \mathrm{NC}$ subjects were finally collected for analysis, who had head motion $<3.0 \mathrm{~mm}$ translation and $3.0^{\circ}$ rotation in any direction during preprocessing of rs-fMRI. Table 1 showed the statistical analysis results of demographics and neuropsychological performance. There was no significant difference between $\mathrm{AD}$ and $\mathrm{NC}$ subjects in demographics $(P>0.05)$. However, there were statistically significant differences in MMSE performance between the two groups $(P<0.05)$.

\section{Functional Connectivity Analysis}

After two-sample $t$-tests, RS FC had no significant difference between $\mathrm{AD}$ and $\mathrm{NC}$ within the left hippocampal mask $(t=0.34, P>0.05)$ and the right hippocampal mask $(t=-1.01, P>0.05)$. In addition, we observed that FC 

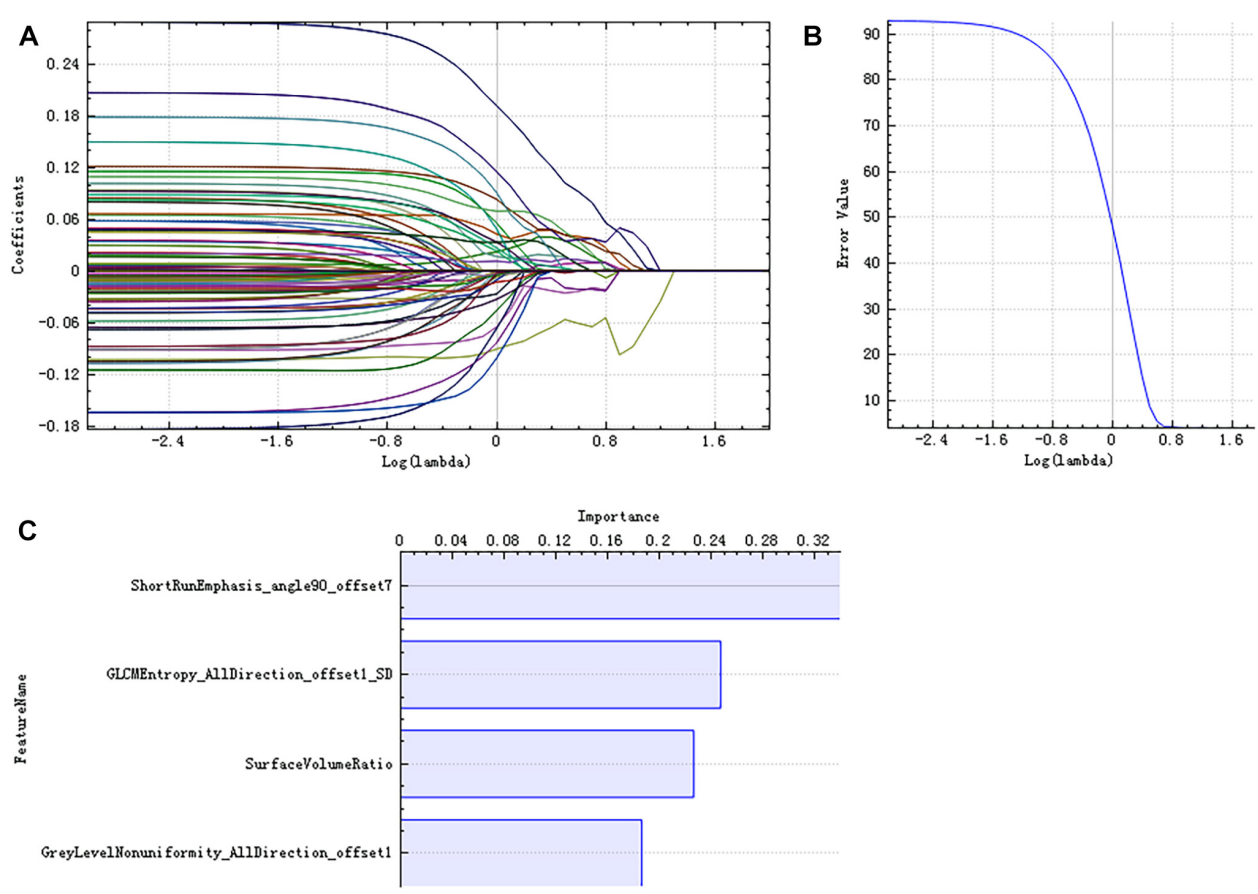

FIGURE 5 | LASSO dimensionality reduction of the right hippocampus. Error-lambda graph (A); coefficients-lambda graph (B). We chose $\lambda$ according to the lowest error rate. Importance of the selected features (C).

A

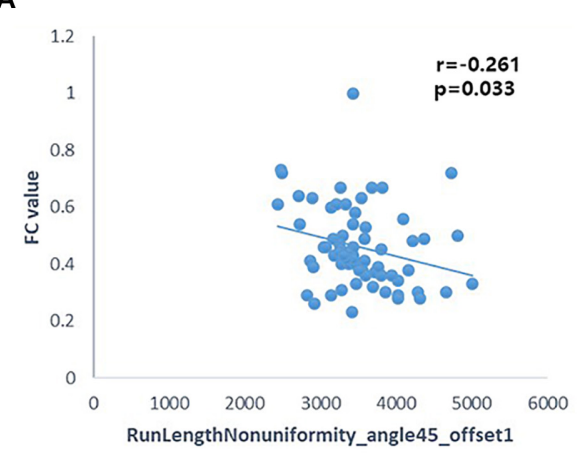

C

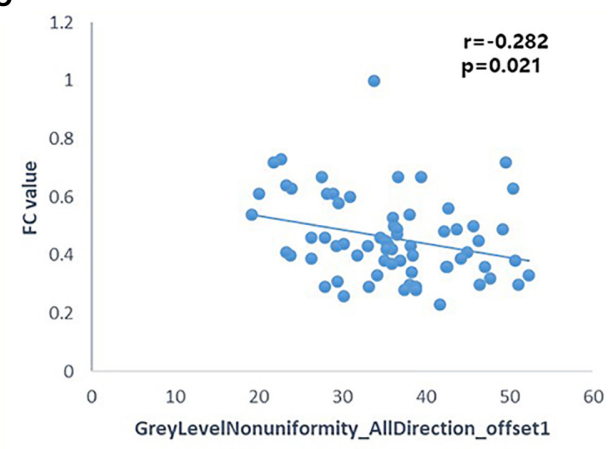

\section{B}

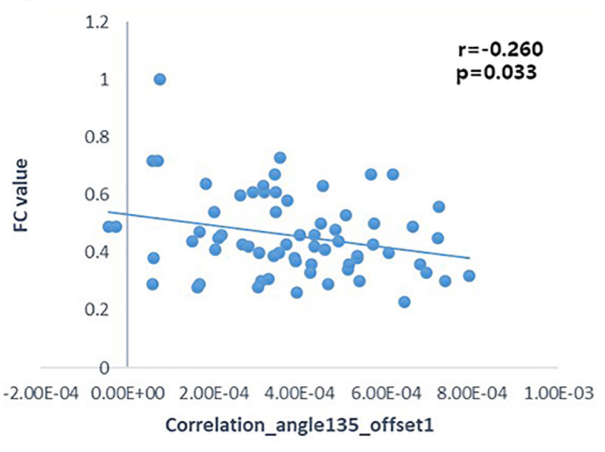

FIGURE 6 | Pearson correlation analysis between some radiomic features and FC of the left hippocampus $(p<0.05)$. Pearson's correlation analysis between "RunLengthNonuniformity_angle45_offset1" and FC value (A); Pearson's correlation analysis between "Correlation_angle135_offset1" and FC value (B); Pearson's correlation analysis between "GreyLevelNonuniformity_AllDirection_offset1" and FC value (C). 
in some brain regions was disrupted in the left hippocampal functional network in the $\mathrm{AD}$ patient group; these regions are right gyrus rectus, right anterior cingulate and paracingulate gyri, right orbital part of middle frontal gyrus, right caudate nucleus, left and right precuneus, left median cingulate and paracingulate gyri, left and right angular gyrus, left and right middle occipital gyrus, and inferior parietal but supramarginal and angular gyri (Figure $\mathbf{1}$ and Table 2). We also observed some brain regions with decreased FC in the right hippocampal functional network, including left and right anterior cingulate and paracingulate gyri, right median cingulate and paracingulate gyri, right dorsolateral superior frontal gyrus, right precentral gyrus, and right middle frontal gyrus (Figure 2 and Table 3 ).

\section{Radiomic Analysis}

There were 385 features extracted in the bilateral hippocampus after feature calculation. For left and right hippocampus, after $T$-test and rank sum test, the remaining feature numbers were 196 and 215. After correlation analysis, the remaining feature numbers were reduced to 70 and 81 (Figures 3A,B). Finally, using the LASSO regression model, five and four features were selected (Figures 4A-C, 5A-C).

\section{Correlations Between Radiomic Features and Functional Connectivity}

Pearson's correlation analysis suggested that there were three radiomic features (RunLengthNonuniformity_angle45_offset1,

TABLE 4 | Correlation between the left hippocampal radiomic features and functional connectivity values.

\begin{tabular}{lcc}
\hline & $\begin{array}{c}\text { Type of } \\
\text { parameters }\end{array}$ & $\begin{array}{c}\text { r, AD patients } \\
(\boldsymbol{n}=\mathbf{6 7})\end{array}$ \\
\hline RunLengthNonuniformity_angle45_offset1 & RLM & $-0.261^{*}$ \\
LowGreyLevelRunEmphasis_AllDirection_offset1 & RLM & 0.222 \\
LowGreyLevelRunEmphasis_AllDirection_offset7_SD & RLM & -0.008 \\
Correlation_angle135_offset1 & GLCM & $-0.260^{*}$ \\
GreyLevelNonuniformity_AllDirection_offset1 & RLM & $-0.282^{*}$ \\
\hline
\end{tabular}

Pearson correlation analyses were conducted to calculate the correlations between these selected features and the left hippocampal FC values. ${ }^{*} P<0.05$. RLM, runlength matrix; GLCM, gray level co-occurrence matrix.

TABLE 5 | Correlation between the right hippocampal radiomic features and functional connectivity values.

\begin{tabular}{lcc}
\hline & $\begin{array}{c}\text { Type of } \\
\text { parameters }\end{array}$ & $\begin{array}{c}\text { r, AD patients } \\
(\boldsymbol{n}=\mathbf{6 7})\end{array}$ \\
\hline ShortRunEmphasis_angle90_offset7 & RLM & 0.203 \\
GLCMEntropy_AllDirection_offset1_SD & GLCM & 0.104 \\
SurfaceVolumeRatio & Formfactor & 0.174 \\
GreyLevelNonuniformity_AllDirection_offset1 & RLM & -0.044
\end{tabular}

Pearson correlation analyses were conducted to calculate the correlations between these selected features and the right hippocampal FC values.
Correlation_angle135_offset1, GreyLevelNonuniformity_AllDirection_offset1) associated with FC values in the left hippocampus $(P<0.05)$; the correlation coefficient values were -0.261 , -0.260 , and -0.282 , respectively (Figure 6 and Table 4). However, there were no significant correlations between selected radiomic features and FC values in the right hippocampus (Table 5).

\section{DISCUSSION}

Our study indicated that the AD group showed abnormalities in the left and right hippocampal functional network compared with the NC group. Meanwhile, the present study selected the closely related radiomic features of the bilateral hippocampus. Pearson correlation analysis suggested weak relationship between some radiomic features and FC values in the left hippocampus. It's a prospective paper to study the correlation between the hippocampal radiomic features and fMRI characteristics in AD.

In addition to hippocampal structural studies, some rsfMRI studies suggest that hippocampal functional characteristics changed in the AD or aMCI stage. For example, Sorg et al. (2007) found that only selected brain areas such as the hippocampus showed reduced activities in MCI patients. Some $\mathrm{AD}$ and MCI studies indicated the destruction of functional connections between the hippocampus and the PCC, medial prefrontal cortex, inferior parietal lobule, and other brain areas (Wang et al., 2006a; Dennis and Thompson, 2014; Krajcovicova et al., 2014). One study showed alterations of three hippocampal subfield functional networks in aMCI patients (De et al., 2017). Our results have demonstrated diminished FC in the right gyrus rectus, right anterior cingulate and paracingulate gyri, bilateral precuneus, bilateral angular gyrus, and bilateral middle occipital gyrus of the left hippocampus functional networks. We also observed some brain regions of decreased FC in the right hippocampal functional network, including bilateral anterior cingulate and paracingulate gyri, right dorsolateral superior frontal gyrus, and right precentral gyrus. Some of these brain regions are major components of the DMN. Most seed-based and ICA studies have shown that functional connections of DMN and other brain networks are reduced in AD or MCI (Greicius et al., 2004; Christian et al., 2007). We observed no increase or decrease of FC in PCC; maybe there are both disruption and compensation effects in the AD stage. However, many studies have reported areas of aberrant increased connectivity in $\mathrm{AD}$ (Damoiseaux et al., 2012; Simona et al., 2015; Li M. et al., 2017). An ICA study by Damoiseaux et al. (2012) showed that the FC of posterior DMN began to decrease in the early stage of $\mathrm{AD}$ patients, while the connection increased within the anterior and ventral DMN. As the disease progressed, all Internet connections decreased. We observed most of the regions of decreased FC in the bilateral hippocampal functional networks, probably because patients with severe $\mathrm{AD}$ were in the majority of our $\mathrm{AD}$ subjects. Moreover, the disruption patterns of the left and right hippocampal networks are 
different in $\mathrm{AD}$ and aMCI patients (Wang et al., 2006b; Xie et al., 2013). Our result was consistent with these recent discoveries.

Radiomics analysis has been applied to some neuropsychiatric diseases. A radiomics study about autism spectrum disorder found significant differences in the texture features in the right hippocampus, corpus callosum, cerebellar white matter, and left choroid plexus between patients and controls (Chaddad et al., 2017b). Some texture analysis studies have found that there are texture differences in hippocampus, corpus callosum, and thalamus between AD patients and NCs (Li et al., 2010; Oliveira et al., 2011). The nine radiomic features selected in this study reflect the differences in image gray value distribution, texture characteristics, spatial heterogeneity, and other microstructural information in AD patients. Among the five selected features of the left hippocampus, "RunLengthNonuniformity_angle45_ offset1," "LowGreyLevelRunEmphasis_AllDirection_offset1," "LowGreyLevel RunEmphasis_AllDirection_offset7_SD," and "GreyLevelNonuniformity_AllDirection_offset1" are RLM parameters. The higher the value of "RunLengthNonuniformity" or "GreyLevelNonuniformity" is, the more heterogeneous the lesion is. "LowGreyLevelRunEmphasis" describes the overall brightness of the lesion; the higher the value, the darker the lesion. "Correlation_angle135_offset1" is one of the GLCM parameters. "Correlation" describes the similarity of the gray levels in adjacent pixels and displays the correlation between a pixel and its neighbors across the image. Among the four selected features of the right hippocampus, "ShortRunEmphasis_angle90_offset7" and "GreyLevelNonuniformity_AllDirection_offset1" are RLM parameters. "ShortRunEmphasis" describes the degree of difference in gray value between adjacent pixels of the lesion; the higher the value is, the more complex and heterogeneous the lesion is. "GLCMEntropy_AllDirection_offset1_SD" is a GLCM parameter. "Entropy" describes the complexity of the co-occurrence matrix; the larger the value is, the more complex the co-occurrence matrix will be. "SurfaceVolumeRatio" is a Formfactor parameter; it describes the three-dimensional size and shape of the hippocampus. If the edge irregularity of three-dimensional lesions is large, the ratio is also large, indicating greater heterogeneity. These features extracted from the hippocampus structure reflect high-order imaging patterns and heterogeneity characteristics of microstructure in hippocampus in $\mathrm{AD}$ patients.

Although there was no significant difference in hippocampal FC between the two groups, there were significant differences in hippocampal radiomic features. In addition, our study showed weak and negative correlation between the intrahippocampal FC and the radiomic features. It suggests that the changes of hippocampal microstructure appeared before the changes of hippocampal function in $\mathrm{AD}$ patients. MR volumetry and DTI studies indicated the decreased volumes and increased mean diffusivity of the hippocampus in AD patients (Palesi et al., 2011; Brueggen et al., 2015). Structural MRI studies have shown that microstructural abnormalities of the hippocampus can be the neuroimaging biomarkers of early cognitive impairment. In future studies, we will pay more attention to the study of hippocampal microstructure to provide imaging basis for the early diagnosis of $\mathrm{AD}$. The occurrence and development of $\mathrm{AD}$ is a complex process, and we can obtain more structural and functional information by using a variety of MRI techniques in future studies.

However, there were several limitations in our study. Firstly, a complete 1:1 match in age and sex ratio had not been achieved. Secondly, although patients with MCI were excluded, the severity of the disease in $\mathrm{AD}$ patients was not distinguished in the study, and these subjects were not followed up. Mild, moderate, and severe $\mathrm{AD}$ patients were all included in the $\mathrm{AD}$ group. Lastly, we did not correct the between-group comparison of RS FC adjusting for hippocampal volume. It may influence the results to a certain degree.

In summary, this study observed that there are decreased activity in hippocampus functional network in AD patients. It also indicates that the closely related hippocampal radiomic features can be neuroimaging biomarkers for the diagnosis of AD. Moreover, we explored the correlations between the MRI radiomic features and intrahippocampal $\mathrm{FC}$ in $\mathrm{AD}$ patients. It provides a very important reference for further understanding the pathogenesis of AD.

\section{ETHICS STATEMENT}

This study was carried out in accordance with the recommendations of "Ethics Committee of Zhejiang Provincial People's Hospital (No. 2012KY002)" with written informed consent from all subjects. All subjects gave written informed consent in accordance with the Declaration of Helsinki. The protocol was approved by the "Ethics Committee of Zhejiang Provincial People's Hospital.”

\section{AUTHOR CONTRIBUTIONS}

$\mathrm{ZD}$, EY, and QF designed the experiments. QF, MW, QS, and $\mathrm{HJ}$ performed the experiments and analyzed the data. ZW segmented the MR images. ZD, QF, PP, and $\mathrm{ZL}$ interpreted the results and drafted the manuscript. All the authors read and approved the final version of the manuscript.

\section{FUNDING}

This study was funded by the National Natural Science Foundation of China (No. 81871337).

\section{ACKNOWLEDGMENTS}

We thank Prof. Wenli Cai for his thoughtful comments and assistance with English editing. 


\section{REFERENCES}

Alderson, T., Kehoe, E., Maguire, L., Farrell, D., Lawlor, B., Kenny, R. A., et al. (2017). Disrupted thalamus white matter anatomy and posterior default mode network effective connectivity in amnestic mild cognitive impairment. Front. Aging Neurosci. 9:370. doi: 10.3389/fnagi.2017.00370

Amp, A. S. (2011). The diagnosis of mild cognitive impairment due to Alzheimer's disease: recommendations from the national institute on aging-Alzheimer's association workgroups on diagnostic guidelines for Alzheimer's disease. Alzheimers Dement. 7, 270-279.

Ball, M. J. (1997). Frequency of stages of Alzheimer-related lesions in different age categories: concurrences and cautions. Neurobiol. Aging 18, 375-376. doi: 10.1016/s0197-4580(97)00052-3

Brueggen, K., Dyrba, M., Barkhof, F., Hausner, L., Filippi, M., Nestor, P. J., et al. (2015). Basal forebrain and hippocampus as predictors of conversion to Alzheimer's disease in patients with mild cognitive impairment-a multicenter DTI and volumetry study. Alzheimers Dement. J. Alzheimers Assoc. 11, 72-72. doi: $10.3233 / J A D-150063$

Bruno, D., Feldman, H. H., Claudia, J., Dekosky, S. T., Pascale, B. G., Jeffrey, C., et al. (2007). Research criteria for the diagnosis of Alzheimer's disease: revising the NINCDS-ADRDA criteria. Lancet Neurol. 6, 734-746. doi: 10.1016/s14744422(07)70178-3

Chaddad, A., Desrosiers, C., Hassan, L., and Tanougast, C. (2017a). Hippocampus and amygdala radiomic biomarkers for the study of autism spectrum disorder. BMC Neurosci. 18:52. doi: 10.1186/s12868-017-0373-0

Chaddad, A., Desrosiers, C., and Toews, M. (2017b). Multi-scale radiomic analysis of sub-cortical regions in MRI related to autism, gender and age. Sci. Rep. 7:45639. doi: 10.1038/srep45639

Christian, S., Valentin, R., Mark, M., Calhoun, V. D., Tom, E., Leonhard, L. E., et al. (2007). Selective changes of resting-state networks in individuals at risk for Alzheimer's disease. Proc. Natl. Acad. Sci. U.S.A. 104, 18760-18765. doi: 10.1073/pnas.0708803104

Claudia, P., Teipel, S. J., Annahita, O., Christian, B. H., Thomas, M., Janaina, M. M., et al. (2010). Automated detection of brain atrophy patterns based on MRI for the prediction of Alzheimer's disease. Neuroimage 50, 162-174. doi: 10.1016/j.neuroimage.2009.11.046

Damoiseaux, J. S., Prater, K. E., Miller, B. L., and Greicius, M. D. (2012). Functional connectivity tracks clinical deterioration in Alzheimer's disease. Neurobiol. Aging 33:828.e19-30

De, F. R., La, J. R., and Chételat, G. (2015). Structural imaging of hippocampal subfields in healthy aging and Alzheimer's disease. Neuroscience 309, 29-50. doi: 10.1016/j.neuroscience.2015.08.033

De, F. R., Mutlu, J., Bejanin, A., Gonneaud, J., Landeau, B., Tomadesso, C., et al. (2017). Intrinsic connectivity of hippocampal subfields in normal elderly and mild cognitive impairment patients. Hum. Brain Mapp. 38, 4922-4932. doi: 10.1002/hbm. 23704

Denise, H., Buckner, R. L., Shimony, J. S., Williams, L. E., Erbil, A., Conturo, T. E., et al. (2004). Differential vulnerability of anterior white matter in nondemented aging with minimal acceleration in dementia of the Alzheimer type: evidence from diffusion tensor imaging. Cereb. Cortex 14, 410-423. doi: 10.1093/cercor/ bhh003

Dennis, E. L., and Thompson, P. M. (2014). Functional brain connectivity using fMRI in aging and Alzheimer's disease. Neuropsychol. Rev. 24, 49-62. doi: 10.1007/s11065-014-9249-6

Dubois, B., Feldman, H. H., Jacova, C., Dekosky, S. T., Barberger-Gateau, P., Cummings, J., et al. (2007). Research criteria for the diagnosis of Alzheimer's disease: revising the NINCDS-ADRDA criteria. Lancet Neurol. 6, 734-746. doi: 10.1016/s1474-4422(07)70178-3

Galloway, M. M. (1975). Texture analysis using gray level run lengths. Comput. Graphics Image Proc. 4, 172-179. doi: 10.1016/s0146-664x(75)80008-6

Greicius, M. D., Gaurav, S., Reiss, A. L., and Vinod, M. (2004). Default-mode network activity distinguishes Alzheimer's disease from healthy aging: evidence from functional MRI. Proc. Natl. Acad. Sci. U.S.A. 101, 4637-4642. doi: 10. 1073/pnas.0308627101

Guzman, V. A., Carmichael, O. T., Schwarz, C., Tosto, G., Zimmerman, M. E., and Brickman, A. M. (2013). White matter hyperintensities and amyloid are independently associated with entorhinal cortex volume among individuals with mild cognitive impairment. Alzheimers Dement. J. Alzheimers Assoc. 9, S124-S131. doi: 10.1016/j.jalz.2012.11.009

Horvat, N., Veeraraghavan, H., Khan, M., Blazic, I., Zheng, J., Capanu, M., et al. (2018). MR imaging of rectal cancer: radiomics analysis to assess treatment response after neoadjuvant therapy. Radiology 287:172300. doi: 10.1148/radiol. 2018172300

Kickingereder, P., Burth, S., Wick, A., Götz, M., Eidel, O., Schlemmer, H. P., et al. (2016). Radiomic profiling of glioblastoma: identifying an imaging predictor of patient survival with improved performance over established clinical and radiologic risk models. Radiology 280, 880-889. doi: 10.1148/radiol. 2016160845

Krajcovicova, L., Marecek, R., Mikl, M., and Rektorova, I. (2014). Disruption of resting functional connectivity in Alzheimer's patients and at-risk subjects. Curr. Neurol. Neurosci. Rep. 14:491. doi: 10.1007/s11910-014-0491-3

Li, X., Xia, H., Zhou, Z., and Tong, L. (2010). “3D texture analysis of hippocampus based on MR images in patients with Alzheimer disease and mild cognitive impairment," in Proceedings of the 2010 3rd International Conference on Biomedical Engineering and Informatics, (Yantai: IEEE).

Li, H., Zhu, Y., Burnside, E. S., Drukker, K., Hoadley, K. A., Fan, C., et al. (2017). MR imaging radiomics signatures for predicting the risk of breast cancer recurrence as given by research versions of mammaprint, oncotype DX, and PAM50 gene assays. Radiology 281:152110.

Li, M., Zheng, G., Zheng, Y., Xiong, Z., Xia, R., Zhou, W., et al. (2017). Alterations in resting-state functional connectivity of the default mode network in amnestic mild cognitive impairment: an fMRI study. BMC Med. Imaging 17:48. doi: 10.1186/s12880-017-0221-9.

Lu, C. F., Hsu, F. T., Hsieh, K. L., Kao, Y. J., Cheng, S. J., Hsu, J. B., et al. (2018). Machine learning-based radiomics for molecular subtyping of gliomas. Clin. Cancer Res. Clincanres. 24, 4429-4436. doi: 10.1158/1078-0432.CCR-173445

Mak, E., Gabel, S., Su, L., Williams, G. B., Arnold, R., Passamonti, L., et al. (2017). Multi-modal MRI investigation of volumetric and microstructural changes in the hippocampus and its subfields in mild cognitive impairment, Alzheimer's disease, and dementia with Lewy bodies. Int. Psychogeriatr. 29, 545-555. doi: $10.1017 /$ S1041610216002143

Menon, V. (2011). Large-scale brain networks and psychopathology: a unifying triple network model. Trends Cognit. Sci. 15, 483-506. doi: 10.1016/j.tics.2011. 08.003

Oliveira, M. S., De Balthazar, M. L. F., D’Abreu, A., Yasuda, C. L., Damasceno, B. P., et al. (2011). MR imaging texture analysis of the corpus callosum and thalamus in amnestic mild cognitive impairment and mild Alzheimer disease. Ajnr. Am. J. Neuroradiol. 32, 60-66. doi: 10.3174/ajnr.A2232

Palesi, F., Vitali, P., Chiarati, P., Castellazzi, G., Caverzasi, E., Pichiecchio, A., et al. (2011). DTI and MR volumetry of hippocampus-PC/PCC circuit: in search of early micro- and macrostructural signs of Alzheimer's disease. Neurol. Res. Int. 2012:517876. doi: 10.1155/2012/517876

Port, J. D. (2018). Diagnosis of attention deficit hyperactivity disorder by using MR imaging and radiomics: a potential tool for clinicians. Radiology 287:631-632. doi: 10.1148/radiol.2018172804

Qi, F., Yuanjun, C., Zhengluan, L., Hongyang, J., Dewang, M., Mei, W., et al. (2018). Corpus callosum radiomics-based classification model in Alzheimer's disease: a Case-control study. Front. Neurol. 9:618. doi: 10.3389/fneur.2018.00618

Seongjin, P., Bohyoung, K., Jeongjin, L., Mo, G. J., and Yeong-Gil, S. (2011). GGO nodule volume-preserving nonrigid lung registration using GLCM texture analysis. IEEE Trans. Biomed. Eng. 58, 2885-2894. doi: 10.1109/TBME.2011. 2162330

Sheline, Y. I., and Raichle, M. E. (2013). Resting state functional connectivity in preclinical Alzheimer's disease. Biol. Psychiatry 74, 340-347. doi: 10.1093/ cercor/bhu259

Shi-Jiang, L., Zhu, L., Gaohong, W., Mei-Jie, Z., Malgorzata, F., and Antuono, P. G. (2002). Alzheimer disease: evaluation of a functional MR imaging index as a marker. Radiology 225, 253-259. doi: 10.1148/radiol.2251011301

Simona, G., Annalena, V., Fabio, S., Fernando, C., Fabrizio, F., Massimo, M., et al. (2015). Increased functional connectivity in the default mode network in mild cognitive impairment: a maladaptive compensatory mechanism associated with poor semantic memory performance. J. Alzheimers Dis. 45, 457-470. doi: 10.3233/JAD- 142547 
Sorg, C., Riedl, V., Mühlau, M., Calhoun, V. D., Eichele, T., Läer, L., et al. (2007). Selective changes of resting-state networks in individuals at risk for Alzheimer's disease. Proc. Natl. Acad. Sci. U.S.A. 104, 18760-18765. doi: 10.1073/pnas. 0708803104

Sun, R., Limkin, E. J., Vakalopoulou, M., Dercle, L., Champiat, S., Han, S. R., et al. (2018). A radiomics approach to assess tumour-infiltrating CD8 cells and response to anti-PD-1 or anti-PD-L1 immunotherapy: an imaging biomarker, retrospective multicohort study. Lancet Oncol. 19, 1180-1191. doi: 10.1016/ S1470-2045(18)30413-3

Toussaint, P. J., Maiz, S., Coynel, D., Doyon, J., Messé, A., Souza, L. C. D., et al. (2014). Characteristics of the default mode functional connectivity in normal ageing and Alzheimer's disease using resting state fMRI with a combined approach of entropy-based and graph theoretical measurements. Neuroimage 101, 778-786. doi: 10.1016/j.neuroimage.2014.08.003

Wang, G., He, L., Yuan, C., Huang, Y., Liu, Z., and Liang, C. (2018). Pretreatment MR imaging radiomics signatures for response prediction to induction chemotherapy in patients with nasopharyngeal carcinoma. Eur. J. Radiol. 98, 100-106. doi: 10.1016/j.ejrad.2017.11.007

Wang, L., Zang, Y., He, Y., Liang, M., Zhang, X., Tian, L., et al. (2006a). Changes in hippocampal connectivity in the early stages of Alzheimer's disease: evidence from resting state fMRI. Neuroimage 31, 496-504. doi: 10.1016/j.neuroimage. 2005.12.033

Wang, L., Zang, Y., Liang, M., Zhang, X., Tian, L., and Jiang, T. (2006b). Changes in hippocampal connectivity in the early stages of Alzheimer's disease: evidence from resting state fMRI. Neuroimage 31, 496-504. doi: 10.1016/j.neuroimage. 2005.12.033

Wang, Z., Yan, C., Zhao, C., Qi, Z., Zhou, W., Lu, J., et al. (2011). Spatial patterns of intrinsic brain activity in mild cognitive impairment and Alzheimer's disease: a resting-state functional MRI study. Hum. Brain Mapp. 32, 1720-1740. doi: 10.1002/hbm. 21140

Whitwell, J. L., Przybelski, S. A., Weigand, S. D., Knopman, D. S., Boeve, B. F., Petersen, R. C., et al. (2007). 3D maps from multiple MRI illustrate changing atrophy patterns as subjects progress from mild cognitive impairment to
Alzheimer's disease. Brain J. Neurol. 130(Pt 7), 1777-1786. doi: 10.1093/brain/ awm 112

Wu, X., Li, R., Fleisher, A. S., Reiman, E. M., Guan, X., Zhang, Y., et al. (2011). Altered default mode network connectivity in Alzheimer's disease-a resting functional MRI and Bayesian network study. Hum. Brain Mapp. 32, 1868-1881. doi: 10.1002/hbm. 21153

Wu, Z., Gao, Y., Shi, F., Ma, G., Jewells, V., and Shen, D. (2017). Segmenting hippocampal subfields from 3T MRI with multi-modality images. Med. Image Anal. 43, 10-22. doi: 10.1016/j.media.2017.09.006

Xie, C., Li, W., Chen, G., Ward, B. D., Franczak, M. B., Jones, J. L., et al. (2013). Late-life depression, mild cognitive impairment and hippocampal functional network architecture. Neuroimage Clin. 3, 311-320. doi: 10.1016/j.nicl.2013. 09.002

Yong, L., Chunshui, Y., Xinqing, Z., Jieqiong, L., Yunyun, D., Alexander-Bloch, A. F., et al. (2014). Impaired long distance functional connectivity and weighted network architecture in Alzheimer's disease. Cereb. Cortex 24, 1422-1435. doi: 10.1093/cercor/bhs410

Yu, E., Liao, Z., Mao, D., Zhang, Q., Ji, G., Li, Y., et al. (2016). Directed functional connectivity of posterior cingulate cortex and whole brain in Alzheimer's disease and mild cognitive impairment. Curr. Alzheimer Res. 14, 628-635. doi: $10.2174 / 1567205013666161201201000$

Conflict of Interest Statement: The authors declare that the research was conducted in the absence of any commercial or financial relationships that could be construed as a potential conflict of interest.

Copyright (c) 2019 Feng, Wang, Song, Wu, Jiang, Pang, Liao, Yu and Ding. This is an open-access article distributed under the terms of the Creative Commons Attribution License (CC BY). The use, distribution or reproduction in other forums is permitted, provided the original author(s) and the copyright owner(s) are credited and that the original publication in this journal is cited, in accordance with accepted academic practice. No use, distribution or reproduction is permitted which does not comply with these terms. 